\title{
Universal form of the Nicolai map
}

\author{
Olaf Lechtenfeld $\odot$ and Maximilian Rupprecht॰ \\ Institut für Theoretische Physik and Riemann Center for Geometry and Physics, \\ Leibniz Universität Hannover, Appelstraße 2, 30167 Hannover, Germany
}

(Received 7 April 2021; accepted 3 June 2021; published 7 July 2021)

\begin{abstract}
The nonlocal bosonic theory obtained from integrating out all anticommuting and auxiliary variables in a globally supersymmetric theory is characterized by the Nicolai map. The latter is generated by a coupling flow functional differential operator, which can be canonically constructed when the supersymmetry is realized off shell. Given any scalar superfield theory, we present a universal formula for both the Nicolai map and its inverse in terms of an ordered exponential of the integrated coupling flow operator. We demonstrate that our formula also holds for supersymmetric gauge theories.
\end{abstract}

DOI: 10.1103/PhysRevD.104.L021701

\section{DEFINITION OF THE NICOLAI MAP}

Supersymmetric theories are normally formulated with bosonic and fermionic (and sometimes ghost) degrees of freedom or, better, using commuting fields $\phi$ and anticommuting fields $\psi$. Since the latter usually occur only quadratically in the action, they are easily integrated out to produce a functional determinant. This leaves one with a purely bosonic but nonlocal theory, given by an action

$$
S_{g}[\phi]=S_{g}^{b}[\phi]+\hbar S_{g}^{f}[\phi],
$$

where $g$ is any coupling constant, and $S_{g}^{b}$ and $S_{g}^{f}$ denote the local and nonlocal parts of the new action, respectively. The latter is proportional to the logarithm of the functional determinant and is down by a factor of $\hbar$ compared to $S_{g}^{b}$, as it generates the fermionic loop contributions to expectation values $^{1}$

$$
\langle X[\phi]\rangle_{g}=\int \mathcal{D} \phi \exp \left\{\frac{\mathrm{i}}{\hbar} S_{g}[\phi]\right\} X[\phi]
$$

of bosonic observables $X[\phi]$. We assume supersymmetry to be unbroken and nonanomalous, so that the vacuum energy vanishes, meaning that $\langle\mathbb{1}\rangle_{g}=1$ and our expectation values are already properly normalized. We leave the spacetime dimension $d$ arbitrary and simply write $\mathrm{d} x$ for its volume element.

\footnotetext{
${ }^{1}$ We work in Minkowski space, but it is also possible to repeat the whole analysis in Euclidean space.

Published by the American Physical Society under the terms of the Creative Commons Attribution 4.0 International license. Further distribution of this work must maintain attribution to the author(s) and the published article's title, journal citation, and DOI. Funded by SCOAP ${ }^{3}$.
}

In 1980 Hermann Nicolai raised and answered the question of how the nonlocal theory $S_{g}$ remembers its supersymmetric heritage [1-3] (see also [4] for a pedagogical introduction). Among all such nonlocal bosonic theories, the ones originating with a supersymmetric past are characterized by the existence of a (nonlinear and nonlocal) field transformation (the Nicolai map)

$$
T_{g}: \phi(x) \mapsto \phi^{\prime}(x ; g, \phi)
$$

invertible at least as a formal power series in $g$, which admits the key identity ${ }^{2}$

$$
\langle X[\phi]\rangle_{g}=\left\langle X\left[T_{g}^{-1} \phi\right]\right\rangle_{0} \quad \forall X,
$$

relating the interacting theory (at coupling $g$ ) to the free one (at coupling $g=0$ ). Writing out the path integrals, this requirement is equivalent to

$$
\begin{aligned}
\mathcal{D} \phi \exp \left\{\frac{\mathrm{i}}{\hbar} S_{g}[\phi]\right\} & =\mathcal{D}\left(T_{g} \phi\right) \exp \left\{\frac{\mathrm{i}}{\hbar} S_{0}\left[T_{g} \phi\right]\right\} \\
& =\mathcal{D} \phi \exp \left\{\frac{\mathrm{i}}{\hbar} S_{0}\left[T_{g} \phi\right]+\operatorname{tr} \ln \frac{\delta T_{g} \phi}{\delta \phi}\right\} .
\end{aligned}
$$

Collecting powers of $\hbar$, one obtains two conditions,

$S_{0}^{b}\left[T_{g} \phi\right]=S_{g}^{b}[\phi] \quad$ and $\quad S_{0}^{f}\left[T_{g} \phi\right]-\mathrm{i} \operatorname{tr} \ln \frac{\delta T_{g} \phi}{\delta \phi}=S_{g}^{f}[\phi]$,

which originally defined the Nicolai map: the local bosonic action is mapped to the free one, and the nonlocal part of

\footnotetext{
${ }^{2}$ This is not the original definition but an equivalent one as we will show shortly.
} 
the action equals the Jacobi determinant of the transformation. We shall henceforth set $\hbar=1$ and only use the relation (4) to construct the Nicolai map below.

\section{COUPLING FLOW OPERATOR}

Except for the rare instances where stochastic variables exist [5-10] the Nicolai map can only be constructed perturbatively. Therefore, it is reasonable to investigate its infinitesimal version. This method was developed in [11-15]. Differentiating (4) with respect to the coupling $g$ yields

$$
\partial_{g}\langle X[\phi]\rangle_{g}=\left\langle\left(\partial_{g}+R_{g}[\phi]\right) X[\phi]\right\rangle_{g}
$$

with a functional differential operator

$$
R_{g}[\phi]=\int \mathrm{d} x\left(\partial_{g} T_{g}^{-1} \circ T_{g}\right) \phi(x) \frac{\delta}{\delta \phi(x)}
$$

that we will refer to as the "coupling flow operator." Its knowledge not only guarantees the existence of the (inverse) Nicolai map but also provides its perturbative construction,

$$
\begin{aligned}
\left(T_{g}^{-1} \phi\right)(x) & =\left.\exp \left\{g\left(\partial_{g^{\prime}}+R_{g^{\prime}}[\phi]\right)\right\} \phi(x)\right|_{g^{\prime}=0} \\
& =\left.\sum_{n=0}^{\infty} \frac{g^{n}}{n !}\left(\partial_{g^{\prime}}+R_{g^{\prime}}[\phi]\right)^{n} \phi(x)\right|_{g^{\prime}=0} .
\end{aligned}
$$

The derivation property of $R_{g}$ is essential to obtain the distributivity of the (inverse) Nicolai map,

$$
T_{g}^{-1} X[\phi]=X\left[T_{g}^{-1} \phi\right] .
$$

Alternatively, the map $T_{g}$ itself may be found iteratively from the relation [15]

$$
\left(\partial_{g}+R_{g}[\phi]\right) T_{g} \phi=0,
$$

which immediately follows from (4) for $X=\phi$.

\section{SCALAR THEORIES}

How to find the coupling flow operator or at least to show its existence, before knowing $T_{g}$ ? If the original local theory in terms of $\phi$ and $\psi$ admits an off-shell supersymmetric formulation, ${ }^{3}$ then its action $S_{\text {SUSY }}$ is the highest component of a superfield, hence can be expressed as a supervariation $\delta_{\alpha}$ of the penultimate component. For scalar supermultiplet theories, the same is true for derivatives with respect to the coupling [11],

$$
\partial_{g} S_{\mathrm{SUSY}}[\phi, \psi]=\delta_{\alpha} \Delta_{\alpha}[\phi, \psi]
$$

\footnotetext{
${ }^{3}$ Auxiliary fields may be kept as part of $\phi$ but it is convenient to integrate them out as well.
}

where $\alpha$ is a Majorana spinor index and $\Delta_{\alpha}$ is a certain anticommuting functional. ${ }^{4}$ Employing (12) and the supersymmetric Ward identity in

$\partial_{g} \int \mathcal{D} \phi \int \mathcal{D} \psi \exp \left\{\mathrm{i}_{\mathrm{SUSY}}[\phi, \psi]\right\} X[\phi]$
$=\mathrm{i} \int \mathcal{D} \phi \int \mathcal{D} \psi \exp \left\{\mathrm{i} S_{\mathrm{SUSY}}[\phi, \psi]\right\}\left(\partial_{g}+\Delta_{\alpha}[\phi, \psi] \delta_{\alpha}\right) X[\phi]$,

we integrate out the anticommuting variables to read off the coupling flow operator

$$
R_{g}[\phi]=\mathrm{i} \underbrace{}_{\alpha}[\phi] \delta_{\alpha}=\mathrm{i} \int \mathrm{d} x \underbrace{\Delta_{\alpha}[\phi]}_{\alpha} \delta_{\alpha} \phi(x) \frac{\delta}{\delta \phi(x)},
$$

where the contraction indicates the presence of a fermionic propagator obtained from a fermionic bilinear.

The main challenge then is to exponentiate this operator in the construction (9). The key new insight here is that the $g^{\prime}$ derivatives on the right-hand side of (9) may actually be performed in closed form, by solving a standard differential equation,

$$
\begin{aligned}
\partial_{g} Z_{g}[\phi] & =Z_{g}[\phi]\left(\partial_{g}+R_{g}[\phi]\right) \\
\Leftrightarrow \quad Z_{g}[\phi] & =Z_{0}[\phi] \stackrel{\mathcal{P}}{\exp \int_{0}^{g} \mathrm{~d} h R_{h}[\phi],}
\end{aligned}
$$

where $\overleftarrow{\mathcal{P}}$ denotes reverse ordering, to be detailed shortly. With the help of the solution $Z_{g}[\phi]$, we obtain

$$
\begin{aligned}
T_{g}^{-1} \phi & =\left.\sum_{n=0}^{\infty} \frac{g^{n}}{n !}\left(Z_{g^{\prime}}[\phi]^{-1} \partial_{g^{\prime}} Z_{g^{\prime}}[\phi]\right)^{n} \phi\right|_{g^{\prime}=0} \\
& =\left.\sum_{n=0}^{\infty} \frac{g^{n}}{n !} Z_{g^{\prime}}[\phi]^{-1} \partial_{g^{\prime}}^{n} Z_{g^{\prime}}[\phi] \phi\right|_{g^{\prime}=0} \\
& =\left.Z_{g^{\prime}}[\phi]^{-1} \mathrm{e}^{g \partial_{g^{\prime}}} Z_{g^{\prime}}[\phi] \phi\right|_{g^{\prime}=0} \\
& =\left.Z_{g^{\prime}}[\phi]^{-1} Z_{g^{\prime}+g}[\phi] \phi\right|_{g^{\prime}=0}=Z_{0}[\phi]^{-1} Z_{g}[\phi] \phi,
\end{aligned}
$$

and therefore

$$
\begin{aligned}
T_{g}^{-1} \phi= & \stackrel{\mathcal{P}}{\exp }\left\{\int_{0}^{g} \mathrm{~d} h R_{h}[\phi]\right\} \phi \\
= & \sum_{s=0}^{\infty} \int_{0}^{g} \mathrm{~d} h_{1} \int_{0}^{h_{1}} \mathrm{~d} h_{2} \ldots \\
& \int_{0}^{h_{s-1}} \mathrm{~d} h_{s} R_{h_{s}}[\phi] \ldots R_{h_{2}}[\phi] R_{h_{1}}[\phi] \phi .
\end{aligned}
$$

\footnotetext{
${ }^{4}$ For gauge theories, which contain vector supermultiplets, the situation is more complicated and will be discussed below.
} 
Apparently, the $g^{\prime}$ derivatives have been traded for integrations, but this representation is more suggestive than (9). Moreover, it allows for an immediate formal inversion to write the Nicolai map itself as

$$
T_{g} \phi=\overrightarrow{\mathcal{P}} \exp \left\{-\int_{0}^{g} \mathrm{~d} h R_{h}[\phi]\right\} \phi=\sum_{s=0}^{\infty}(-1)^{s} \int_{0}^{g} \mathrm{~d} h_{s} \ldots \int_{0}^{h_{3}} \mathrm{~d} h_{2} \int_{0}^{h_{2}} \mathrm{~d} h_{1} R_{h_{s}}[\phi] \ldots R_{h_{2}}[\phi] R_{h_{1}}[\phi] \phi,
$$

with $\overrightarrow{\mathcal{P}}$ indicating standard ordering, This universal form is the main result of our work.

It is instructive to express the power series expansions of $T_{g}^{-1} \phi$ and of $T_{g} \phi$ with the one for the flow operator,

$R_{g}[\phi]=\sum_{k=1}^{\infty} g^{k-1} \mathbf{R}_{k}[\phi]=\mathbf{R}_{1}[\phi]+g \mathbf{R}_{2}[\phi]+g^{2} \mathbf{R}_{3}[\phi]+\ldots$

(the shift in the $g$ power is a practical convention here). With this, the integrals in (17) and (18) can be evaluated to yield

$$
T_{g}^{-1} \phi=\sum_{\mathbf{n}} g^{n} d_{\mathbf{n}} \mathbf{R}_{n_{s}}[\phi] \ldots \mathbf{R}_{n_{2}}[\phi] \mathbf{R}_{n_{1}}[\phi] \phi
$$

and

$$
T_{g} \phi=\sum_{\mathbf{n}} g^{n} c_{\mathbf{n}} \mathrm{R}_{n_{s}}[\phi] \ldots \mathrm{R}_{n_{2}}[\phi] \mathrm{R}_{n_{1}}[\phi] \phi,
$$

respectively, where the boldface letter denotes the multi-index

$\mathbf{n}=\left(n_{1}, n_{2}, \ldots, n_{s}\right) \quad$ with $\quad n_{i} \in \mathbb{N} \quad$ and $\quad \sum_{i} n_{i}=n$,

where $1 \leq s \leq n$ and the $n=0$ term is the identity. The numerical coefficients are computed as

$$
\begin{aligned}
d_{\mathbf{n}} & =\int_{0}^{1} \mathrm{~d} x_{1} x_{1}^{n_{1}-1} \int_{0}^{x_{1}} \mathrm{~d} x_{2} x_{2}^{n_{2}-1} \cdots \int_{0}^{x_{s-1}} \mathrm{~d} x_{s} x_{s}^{n_{s}-1} \\
& =\left[n_{s} \cdot\left(n_{s}+n_{s-1}\right) \cdots\left(n_{s}+n_{s-1}+\cdots+n_{1}\right)\right]^{-1}, \\
c_{\mathbf{n}} & =(-1)^{s} \int_{0}^{1} \mathrm{~d} x_{s} x_{s}^{n_{s}-1} \cdots \int_{0}^{x_{3}} \mathrm{~d} x_{2} x_{2}^{n_{2}-1} \int_{0}^{x_{2}} \mathrm{~d} x_{1} x_{1}^{n_{1}-1} \\
& =(-1)^{s}\left[n_{1} \cdot\left(n_{1}+n_{2}\right) \cdots\left(n_{1}+n_{2}+\cdots+n_{s}\right)\right]^{-1},
\end{aligned}
$$

the latter being in agreement with the earlier result in [15] derived from (11). Writing out the first few terms, the perturbative Nicolai map reads

$$
\begin{aligned}
T_{g} \phi= & \phi-g \mathrm{R}_{1} \phi-\frac{1}{2} g^{2}\left(\mathrm{R}_{2}-\mathrm{R}_{1}^{2}\right) \phi \\
& -\frac{1}{6} g^{3}\left(2 \mathrm{R}_{3}-\mathrm{R}_{1} \mathrm{R}_{2}-2 \mathrm{R}_{2} \mathrm{R}_{1}+\mathrm{R}_{1}^{3}\right) \phi \\
& -\frac{1}{24} g^{4}\left(6 \mathrm{R}_{4}-2 \mathrm{R}_{1} \mathrm{R}_{3}-3 \mathrm{R}_{2} \mathrm{R}_{2}+\mathrm{R}_{1}^{2} \mathrm{R}_{2}\right. \\
& \left.-6 \mathrm{R}_{3} \mathrm{R}_{1}+2 \mathrm{R}_{1} \mathrm{R}_{2} \mathrm{R}_{1}+3 \mathrm{R}_{2} \mathrm{R}_{1}^{2}-\mathrm{R}_{1}^{4}\right) \phi+\mathcal{O}\left(g^{5}\right) .
\end{aligned}
$$

\section{GAUGE THEORIES}

Supersymmetric Yang-Mills theory is a cornerstone of modern mathematical physics and therefore of prime interest. The Nicolai map promises an alternative approach to its quantization and has regained some attention recently [16-19]. Let us hence consider unbroken $\mathcal{N}=1$ supersymmetric gauge theories in the Wess-Zumino gauge, with the field content $(A, \lambda, D)$ in the adjoint representation of the gauge group and the Yang-Mills field strength

$$
F=\mathrm{d} A+g A \wedge A=\frac{1}{2} F_{\mu \nu} \mathrm{d} x^{\mu} \wedge \mathrm{d} x^{\nu} .
$$

Choosing a linear gauge fixing ${ }^{5}$

$$
0=\mathcal{G}(A)=\partial^{\mu} A_{\mu} \quad \text { or } \quad n^{\mu} A_{\mu}
$$

adds, via the Faddeev-Popov trick and the 't Hooft averaging, a gauge-fixing term depending on ghost fields $C$ and $\bar{C}$ and a gauge parameter $\xi$ to the action. This explicitly breaks supersymmetry and reduces the gauge symmetry to Becchi-Rouet-Stora-Tyutin (BRST) invariance. The construction of the Nicolai map now presents an additional challenge, because the $g$ derivative of

$$
\begin{aligned}
S_{\mathrm{SUSY}} & {[A, \lambda, D, C, \bar{C}] } \\
= & \int \mathrm{d} x \operatorname{tr}\left\{-\frac{1}{4} F_{\mu \nu} F^{\mu \nu}-\frac{1}{2 \xi} \mathcal{G}(A)^{2}+\right.\text { fermions } \\
& + \text { ghosts }+ \text { auxiliaries }\}
\end{aligned}
$$

\footnotetext{
${ }^{5}$ For convenience, nonlinear gauges are easily accommodated with $\mathcal{G}(A)=\tilde{\mathcal{G}}(g A) / g$ for an arbitrary function $\tilde{\mathcal{G}}$.
} 
is no longer a supervariation, even not up to a Slavnov variation (which generates the BRST transformations). The way out is a rescaling of all fields with a suitable power of $g$, with the total Jacobian being unity. In particular, for the commuting fields we define

and arrive at

$$
\tilde{A}=g A \quad \Rightarrow \quad \tilde{F}=g F=\mathrm{d} \tilde{A}+\tilde{A} \wedge \tilde{A}=\frac{1}{2} \tilde{F}_{\mu \nu} \mathrm{d} x^{\mu} \wedge \mathrm{d} x^{\nu} \quad \text { and } \quad \tilde{D}=g D
$$

$$
S_{\mathrm{SUSY}}[\tilde{A}, \tilde{\lambda}, \tilde{D}, \tilde{C}, \tilde{\bar{C}}]=\frac{1}{g^{2}} \int \mathrm{d} x \operatorname{tr}\left\{-\frac{1}{4} \tilde{F}_{\mu \nu} \tilde{F}^{\mu \nu}-\frac{1}{2 \xi} \mathcal{G}(A)^{2}+\text { fermions }+ \text { ghosts }+ \text { auxiliaries }\right\}
$$

where the only $g$ dependence resides in front of the integral and in a factor of $g$ multiplying the ghost term. It is then easy to show that $[12,13]$

$$
\begin{gathered}
\partial_{g} S_{\mathrm{SUSY}}=-\frac{1}{g^{3}}\left\{\delta_{\alpha} \Delta_{\alpha}[\tilde{A}, \tilde{\lambda}, \tilde{D}]-\sqrt{g} \Delta_{\mathrm{gh}}[\tilde{\bar{C}}, \tilde{A}]\right\} \\
\text { with } \quad \Delta_{\text {gh }}=\int \operatorname{tr}\{\tilde{\bar{C}} \mathcal{G}(\tilde{A})\}
\end{gathered}
$$

where $\Delta_{\alpha}$ is a particular gauge-invariant fermionic functional and "s" denotes the (anticommuting) Slavnov variation. With this information, we can employ the Ward identities for BRST and broken supersymmetry to compute the effect of a $g$ derivative on the expectation value $\langle X[\tilde{A}]\rangle_{g}$ after integrating out gaugini, ghosts, and auxiliaries,

$$
\partial_{g}\langle X[\tilde{A}]\rangle_{g}=\left\langle\left(\partial_{g}+\frac{1}{g} \tilde{R}[\tilde{A}]\right) X[\tilde{A}]\right\rangle_{g}
$$

where the coupling flow operator is given by $[12,13]$

$$
\begin{aligned}
& \tilde{R}[\tilde{A}]=-\mathrm{i} \Delta_{\alpha[\tilde{A}]} \delta_{\alpha}+\frac{\mathrm{i}}{\sqrt{g}} \underline{\Delta_{\mathrm{gh}}[\tilde{A}] s}-\frac{1}{\sqrt{g}} \underline{\Delta_{\alpha}[\tilde{A}]\left(\delta_{\alpha}\right.} \underline{\left.\Delta_{\mathrm{gh}}[\tilde{A}]\right) s} \\
& \text { with } s \tilde{A}_{\mu}=\sqrt{g} \tilde{D}_{\mu} \widetilde{C},
\end{aligned}
$$

and contractions indicating either gaugino or ghost propagators. It is just a complicated linear functional differential operator. Remarkably, in the variable $\tilde{A}$ it is independent of the gauge coupling $g$. that

Observing that $\Delta_{\text {gh }}[\tilde{A}] s \mathcal{G}(\tilde{A})=-\mathrm{i} \sqrt{g} \mathcal{G}(\tilde{A})$, it follows

$$
\tilde{R}[\tilde{A}] \mathcal{G}(\tilde{A})=\mathcal{G}(\tilde{A}) \quad \Rightarrow \quad\left(\partial_{g}+\frac{1}{g} \tilde{R}[\tilde{A}]\right) \frac{1}{g} \mathcal{G}(\tilde{A})=0,
$$

which implies that the chosen gauge class $\mathcal{G}(A)=\frac{1}{g} \mathcal{G}(\tilde{A})$ is invariant under the coupling constant flow generated by $\tilde{R}$ and hence a fixed point of the Nicolai map. This property is an additional requirement in the usual definition of the Nicolai map [3], but it is automatic here from the construction of $\tilde{R}$.

In order to integrate the coupling flow and obtain the analog of (9) it is necessary to revert the rescaling (29),

$$
T_{g}^{-1} A=\left.\left.\frac{1}{g} \exp \left\{g\left(\partial_{g^{\prime}}+\frac{1}{g^{\prime}} \tilde{R}[\tilde{A}]\right)\right\} \tilde{A}\right|_{\tilde{A}=g^{\prime} A}\right|_{g^{\prime}=0} .
$$

It is not manifest but true that the final step $g^{\prime} \rightarrow 0$ is nonsingular. The $g^{\prime}$ derivatives in the exponent can be executed,

$$
\begin{aligned}
T_{g}^{-1} A & =\left.\left.\frac{1}{g} \sum_{n=0}^{\infty} \frac{g^{n}}{n !}\left(\partial_{g^{\prime}}+\frac{1}{g^{\prime}} \tilde{R}[\tilde{A}]\right)^{n} \tilde{A}\right|_{\tilde{A}=g^{\prime} A}\right|_{g^{\prime}=0}, \\
& =\left.\left.\frac{1}{g} \sum_{n=0}^{\infty} \frac{g^{n}}{n !}\left(\left(g^{\prime}\right)^{-\tilde{R}[\tilde{A}]} \partial_{g^{\prime}}\left(g^{\prime}\right)^{\tilde{R}[\tilde{A}]}\right)^{n} \tilde{A}\right|_{\tilde{A}=g^{\prime} A}\right|_{g^{\prime}=0}, \\
& =\left.\left.\frac{1}{g} \sum_{n=0}^{\infty} \frac{g^{n}}{n !}\left(g^{\prime}\right)^{-\tilde{R}[\tilde{A}]} \partial_{g^{\prime}}^{n}\left(g^{\prime}\right)^{\tilde{R}[\tilde{A}] \tilde{A}}\right|_{\tilde{A}=g^{\prime} A}\right|_{g^{\prime}=0}, \\
& =\left.\left.\frac{1}{g}\left(g^{\prime}\right)^{-\tilde{R}[\tilde{A}]} \exp \left\{g \partial_{g^{\prime}}\right\}\left(g^{\prime}\right)^{\tilde{R}[\tilde{A}] \tilde{A}}\right|_{\tilde{A}=g^{\prime} A}\right|_{g^{\prime}=0}, \\
& =\left.\left.\frac{1}{g}\left(g^{\prime}\right)^{-\tilde{R}[\tilde{A}]}\left(g^{\prime}+g\right)^{\tilde{R}[\tilde{A}] \tilde{A}}\right|_{\tilde{A}=g^{\prime} A}\right|_{g^{\prime}=0}=\left.\frac{g^{\prime}}{g}\left(1+\frac{g}{g^{\prime}}\right)^{\tilde{R}\left[g^{\prime} A\right]} A\right|_{g^{\prime}=0}, \\
& =\left.\sum_{n=1}^{\infty} \frac{1}{n !}\left(\frac{g}{g^{\prime}}\right)^{n-1} \tilde{R}\left[g^{\prime} A\right]\left(\tilde{R}\left[g^{\prime} A\right]-1\right) \cdots\left(\tilde{R}\left[g^{\prime} A\right]-n+1\right) A\right|_{g^{\prime}=0},
\end{aligned}
$$


but regularity at $g^{\prime}=0$ is still not obvious. In order to clarify this property, it is convenient to break up $\tilde{R}$ into homogeneous pieces and split off the degree-zero part (remember $\tilde{A}=g A$ ),

$$
\tilde{R}[\tilde{A}]=\sum_{k=0}^{\infty} \mathbf{R}_{k}[\tilde{A}]=: \mathbf{R}_{0}[A]+g R_{g}[A]
$$

with $\quad E \mathrm{R}_{k}[\tilde{A}] \equiv \int \tilde{A} \frac{\delta}{\delta \tilde{A}} \mathrm{R}_{k}[\tilde{A}]=k \mathrm{R}_{k}[\tilde{A}]$, where we defined the functional Euler operator $E$. When scaling back from $\tilde{A}$ to $A$, it is useful to recall for any functional $F$ the obvious equivalence

$$
g \partial_{g} F[\tilde{A}]=0 \quad \Leftrightarrow \quad\left(g \partial_{g}-E\right) F[g A]=0 .
$$

Applying this in the fourth line below and twice employing $\left[g \partial_{g}, \frac{1}{g}\right]=-\frac{1}{g}$, we obtain

$$
\begin{aligned}
T_{g}^{-1} A & =\left.\left.\frac{1}{g} \sum_{n=0}^{\infty} \frac{g^{n}}{n !}\left[\frac{1}{g^{\prime}}\left(g^{\prime} \partial_{g^{\prime}}+\tilde{R}[\tilde{A}]\right)\right]^{n} \tilde{A}\right|_{\tilde{A}=g^{\prime} A}\right|_{g^{\prime}=0}, \\
& =\left.\left.\frac{1}{g} \sum_{n=1}^{\infty} \frac{1}{n !}\left(\frac{g}{g^{\prime}}\right)^{n}(\tilde{R}[\tilde{A}]-n+1) \cdots(\tilde{R}[\tilde{A}]-1) \tilde{R}[\tilde{A}] \tilde{A}\right|_{\tilde{A}=g^{\prime} A}\right|_{g^{\prime}=0}, \\
& =\left.\frac{1}{g} \sum_{n=1}^{\infty} \frac{1}{n !}\left(\frac{g}{g^{\prime}}\right)^{n}\left(\tilde{R}\left[g^{\prime} A\right]-n+1\right) \cdots\left(\tilde{R}\left[g^{\prime} A\right]-1\right) \tilde{R}\left[g^{\prime} A\right] g^{\prime} A\right|_{g^{\prime}=0}, \\
& =\left.\frac{1}{g} \sum_{n=1}^{\infty} \frac{1}{n !}\left(\frac{g}{g^{\prime}}\right)^{n}\left(g^{\prime} \partial_{g^{\prime}}-E+\tilde{R}\left[g^{\prime} A\right]-n+1\right) \cdots\left(g^{\prime} \partial_{g^{\prime}}-E+\tilde{R}\left[g^{\prime} A\right]\right) g^{\prime} A\right|_{g^{\prime}=0}, \\
& =\left.\frac{1}{g} \sum_{n=1}^{\infty} \frac{g^{n}}{n !}\left[\frac{1}{g^{\prime}}\left(g^{\prime} \partial_{g^{\prime}}-E+\tilde{R}\left[g^{\prime} A\right]\right)\right]^{n} g^{\prime} A\right|_{g^{\prime}=0}, \\
& =\left.\frac{1}{g} \sum_{n=1}^{\infty} \frac{g^{n}}{n !}\left(\partial_{g^{\prime}}+\frac{1}{g^{\prime}}\left(\mathrm{R}_{0}[A]-E\right)+R_{g^{\prime}}[A]\right)^{n} g^{\prime} A\right|_{g^{\prime}=0}, \\
& =\left.\frac{1}{g} \sum_{n=1}^{\infty} \frac{g^{n}}{n !}\left(\partial_{g^{\prime}}+R_{g^{\prime}}[A]\right)^{n} g^{\prime} A\right|_{g^{\prime}=0}=\left.\frac{1}{g} \sum_{n=1}^{\infty} \frac{g^{n}}{n !} n\left(\partial_{g^{\prime}}+R_{g^{\prime}}[A]\right)^{n-1} A\right|_{g^{\prime}=0},
\end{aligned}
$$

where we noticed and used the necessity

$\mathrm{R}_{0}[A]=E=\int A \frac{\delta}{\delta A} \Leftrightarrow T_{g}^{-1} A=A+g \mathrm{R}_{1}[A] A+\mathcal{O}\left(g^{2}\right)$,

which is borne out by explicit computation as well $[15,18]$. Therefore, with the coupling flow operators in the two field scalings being related by

$$
R_{g}[A]=\frac{1}{g}(\tilde{R}[g A]-E),
$$

the final inverse Nicolai map reads

$$
\begin{aligned}
T_{g}^{-1} A & =\left.\sum_{n=0}^{\infty} \frac{g^{n}}{n !}\left(\partial_{g^{\prime}}+R_{g^{\prime}}[A]\right)^{n} A\right|_{g^{\prime}=0} \\
& =\left.\exp \left\{g\left(\partial_{g^{\prime}}+R_{g^{\prime}}[A]\right)\right\} A\right|_{g^{\prime}=0} .
\end{aligned}
$$

This form has been employed directly already in [2,3] for $d=4$ and again in [18] for the critical dimensions $d=3,4,6$ and 10. Appendix A of [18] generalized the earlier proof of existence to all critical dimensions without the need for off shell supersymmetry but only in the Landau gauge.

Obviously, (42) is of the same form as (9) for scalar theories. Therefore, the universal forms (17) and (18) apply for gauge theories as well,

$$
\begin{aligned}
T_{g} A & =\overrightarrow{\mathcal{P}} \exp \left\{-\int_{0}^{g} \mathrm{~d} h R_{h}[A]\right\} A \\
& =\sum_{\mathbf{n}} g^{n} c_{\mathbf{n}} \mathrm{R}_{n_{s}}[A] \ldots \mathrm{R}_{n_{2}}[A] \mathrm{R}_{n_{1}}[A] A .
\end{aligned}
$$

This, together with (41), is our second main result.

\section{CONCLUSIONS AND OUTLOOK}

The property $\langle X[\phi]\rangle_{g}=\left\langle X\left[T_{g}^{-1} \phi\right]\right\rangle_{0}$ suffices to define the inverse Nicolai map $T_{g}^{-1}$. We briefly reviewed how off shell supersymmetry admits the construction of a coupling flow operator $R_{g}[\phi]$, which generates the inverse Nicolai map via exponentiation of $\partial_{g}+R_{g}[\phi]$. The $g$ 
derivatives can be integrated to find a universal formula for the Nicolai map as $T_{g}=\overrightarrow{\mathcal{P}} \exp \left\{-\int_{0}^{g} \mathrm{~d} h R_{h}[\phi]\right\}$ given by an ordered exponential. This formula applies both to scalar and gauge superfield theories, and it recovers the correct power series expansion of $T_{g}$. For gauge theories the coupling flow automatically respects the gauge choice.

Various applications are in sight, namely the gauge dependence and uniqueness of the Nicolai map, the absence of off shell supersymmetry in higher dimensions, nonlinear sigma models, extended supersymmetry, or supersymmetry breaking (which may be triggered by an external field, as for the matrix models in [20]). Since Lorentz invariance is not necessary for our construction, our scope includes non-Lorentzian theories with off shell supersymmetry, such as [21]. We hope to come back to these issues.

\section{ACKNOWLEDGMENTS}

M. R. is supported by a $\mathrm{PhD}$ grant of the German Academic Scholarship Foundation.
[1] H. Nicolai, On a new characterization of scalar supersymmetric theories, Phys. Lett. 89B, 341 (1980).

[2] H. Nicolai, Supersymmetry and functional integration measures, Nucl. Phys. B176, 419 (1980).

[3] H. Nicolai, Supersymmetric functional integration measures, Lectures Delivered at the NATO Advanced Study Institute on Supersymmetry, Bonn, Germany, 20-31 Aug 1984, edited by K. Dietz et al. (Plenum Press, New York, 1984), pp. 393-420.

[4] H. Ezawa and J. Klauder, Fermions without fermions: The Nicolai map revisited, Prog. Theor. Phys. 74, 904 (1985).

[5] V. de Alfaro, S. Fubini, G. Furlan, and G. Veneziano, Stochastic identities in supersymmetric theories, Phys. Lett. 142B, 399 (1984).

[6] V. de Alfaro, S. Fubini, G. Furlan, and G. Veneziano, Stochastic identities in quantum theory, Nucl. Phys. B255, 1 (1985).

[7] V. de Alfaro, S. Fubini, G. Furlan, and G. Veneziano, Nicolai mapping and stochastic identities in supersymmetric field theories, Phys. Rep. 137, 55 (1986).

[8] R. Floreani, J. P. Leroy, J. Michel, and G. C. Rossi, A perturbative study of the Nicolai mapping, Phys. Lett. 158B, 47 (1985).

[9] V. de Alfaro, S. Fubini, and G. Furlan, Stochastic identities in the light cone gauge, Phys. Lett. 163B, 176 (1985).

[10] M. Bochicchio and A. Pilloni, Gauge theories in antiselfdual variables, J. High Energy Phys. 09 (2013) 039.

[11] R. Flume and O. Lechtenfeld, On the stochastic structure of globally supersymmetric field theories, Phys. Lett. 135B, 91 (1984).
[12] K. Dietz and O. Lechtenfeld, Nicolai maps and stochastic observables from a coupling constant flow, Nucl. Phys. B255, 149 (1985).

[13] O. Lechtenfeld, Construction of the Nicolai mapping in supersymmetric field theories, Ph.D. Thesis, Bonn University, 1984 [Internal Report No. BONN-IR-84-42, ISSN-0172-8741].

[14] K. Dietz and O. Lechtenfeld, Ghost-free quantisation of non-Abelian gauge theories via the Nicolai transformation of their supersymmetric extensions, Nucl. Phys. B259, 397 (1985).

[15] O. Lechtenfeld, Stochastic variables in ten dimensions? Nucl. Phys. B274, 633 (1986).

[16] S. Ananth, H. Nicolai, C. Pandey, and S. Pant, Supersymmetric Yang-Mills theories: Not quite the usual perspective, J. Phys. A 53, 174001 (2020).

[17] H. Nicolai and J. Plefka, $\mathcal{N}=4$ super-Yang-Mills correlators without anticommuting variables, Phys. Rev. D 101, 125013 (2020).

[18] S. Ananth, O. Lechtenfeld, H. Malcha, H. Nicolai, C. Pandey, and S. Pant, Perturbative linearization of supersymmetric Yang-Mills theory, J. High Energy Phys. 10 (2020) 199.

[19] S. Ananth, H. Malcha, C. Panday, and A. Pant, Supersymmetric Yang-Mills theory in $D=6$ without anticommuting variables, Phys. Rev. D 103, 025010 (2021).

[20] T. Kuroki and F. Sugino, Spontaneous supersymmetry breaking in matrix models from the viewpoints of localization and Nicolai mapping, Nucl. Phys. B844, 409 (2011).

[21] R. Auzzi, S. Baiguera, G. Nardelli, and S. Penati, Renormalization properties of a Galilean Wess-Zumino model, J. High Energy Phys. 06 (2019) 048. 